\title{
La Red de las Américas para la Equidad en Salud: inclusión, compromiso y acción
}

\author{
Arachu Castro ${ }^{1}$, Rocío Sáenz², Ximena Avellaneda ${ }^{3}$, Carlos Cáceres ${ }^{4}$, Luiz Galvão ${ }^{5}$, Pedro Mas ${ }^{6}$, \\ Amy E. Ritterbusch ${ }^{7}$ y Manuel Urbina Fuentes ${ }^{8}$
}

Forma de citar

Castro A, Sáenz R, Avellaneda X, Cáceres C, Galvão L, Mas P et al. La Red de las Américas para la Equidad en Salud: inclusión, compromiso y acción. Rev Panam Salud Publica. 2020;44:e130. https://doi.org/10.26633/RPSP.2020.130

RESUMEN La Red de las Américas para la Equidad en Salud (RAES) es una red multidisciplinaria que promueve el
intercambio de conocimientos y la acción intersectorial para la equidad en salud y los derechos humanos
en las Américas. Los objetivos de la RAES consisten en: 1) compartir experiencias exitosas en el desarro-
Ilo de intervenciones, considerando la determinación y los determinantes sociales, para lograr respuestas
participativas y comunitarias en salud; 2) analizar los impactos sanitarios, sociales, políticos, ambientales
y económicos de la pandemia de COVID-19; 3) identificar los efectos de la atención de la pandemia en las
poblaciones de mayor riesgo por su edad y las condiciones de salud preexistentes; 4) examinar la situación
de las fronteras y de los movimientos de población en la propagación de la pandemia y de sus efectos en las
poblaciones migrantes; 5) proponer estrategias para asegurar el acceso a la atención integral de las mujeres
gestantes, con el fin de reducir el sufrimiento, la morbilidad y la mortalidad materna y neonatal; y 6 ) analizar
vulneraciones de derechos humanos y del derecho a la salud de poblaciones históricamente marginalizadas,
incluyendo habitantes en situación de calle y otras comunidades que dependen de los espacios públicos y
de la calle para sobrevivir. Los modelos analíticos y de intervención para la equidad en salud de la RAES se
desarrollan desde varios enfoques, como la medicina social, la epidemiologia social, la antropología médica,
la ecología humana y el de Una salud.

Palabras clave Equidad en salud; salud única; colaboración intersectorial; determinantes sociales de la salud; Américas.

\section{QUÉ ES LA RED DE LAS AMÉRICAS PARA LA EQUIDAD EN SALUD}

La Red de las Américas para la Equidad en Salud (RAES) es una red multidisciplinaria cuyo objetivo es promover el intercambio de conocimientos y la acción intersectorial para la equidad en salud y los derechos humanos en las Américas. En una primera etapa, la RAES tuvo su sede en la Universidad de California en Los Ángeles (1). A partir de 2020, la red se estableció en la Universidad de Costa Rica, desde donde forja alianzas con otras entidades académicas y no gubernamentales, sociedades científicas y organizaciones internacionales.

La RAES promueve un modelo multidimensional de análisis: la determinación (2) y los determinantes sociales de la salud $(3,4)$ y del bienestar (5), la salud en todas las políticas (6) y la salud planetaria (7), perspectiva que considera la interacción ponderada entre la humanidad y los sistemas naturales para promocionar la salud, el bienestar y la equidad y alcanzar los Objetivos de Desarrollo Sostenible (ODS) de la Agenda 2030 para el Desarrollo Sostenible $(8,9)$. Para ello es imprescindible

\footnotetext{
1 Escuela de Salud Pública y Medicina Tropical de la Universidad de Tulane, Nueva Orleans, Estados Unidos de América. $\triangle$ acastro1@tulane.edu.

2 Escuela de Salud Pública, Universidad de Costa Rica, San José, Costa Rica

3 Grupo de Estudios sobre la Mujer Rosario Castellanos, Oaxaca, México

4 Facultad de Salud Pública y Administración, Universidad Peruana Cayetano Heredia, Lima, Perú
}

\footnotetext{
5 Fundación Oswaldo Cruz, Rio de Janeiro, Brasil

6 Instituto de Medicina Tropical Pedro Kourí, La Habana, Cuba

Escuela Luskin de Asuntos Públicos, Universidad de California, Los Ángeles, Estados Unidos de América

8 Academia Nacional de Medicina, Ciudad de México, México
} 
FIGURA 1. Marco conceptual del impacto de los determinantes sociales de la salud en la equidad en salud

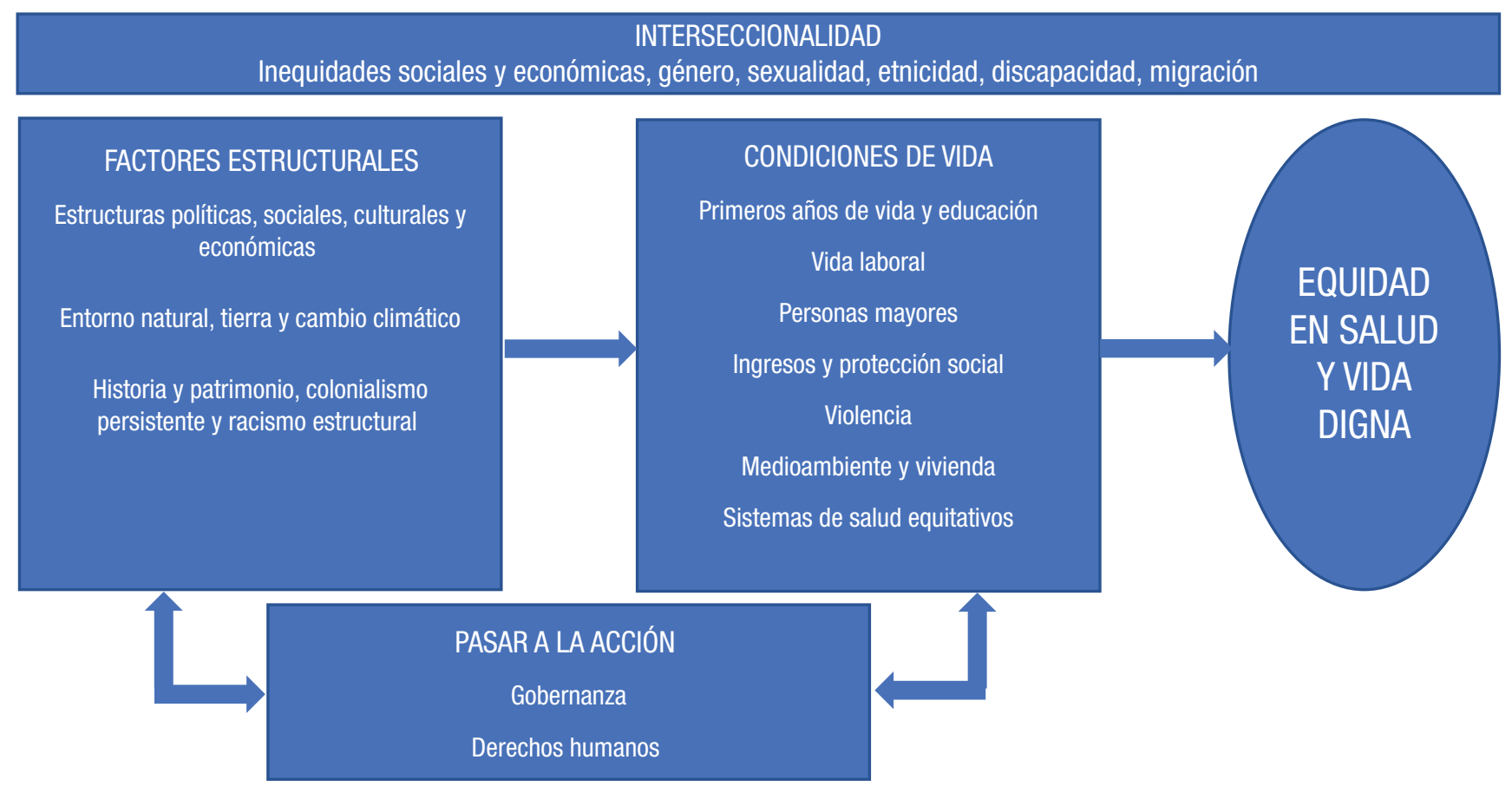

Fuente: Sociedades justas: Equidad en la salud y vida digna. OPS, 2019 (3).

implementar, de forma intersectorial y colaborativa, políticas públicas de salud, educación y protección social y contar con seguridad humana y la preservación del planeta, y se requiere que las dimensiones económica, social y ambiental — que constituyen los determinantes sociales de la salud-se aborden de manera integrada (Figura 1).

La RAES se sustenta en ejes teóricos que incluyen el concepto de equidad en salud para que todas las personas tengan la oportunidad de alcanzar su máximo potencial y que, ante la misma necesidad, tengan igualdad de acceso y de atención de calidad en salud (10), así como en el de inequidad en salud o diferencias en salud que son "sistemáticas, evitables e injustas" (11). Los modelos analíticos y de intervención para la equidad en salud de la RAES se desarrollan desde varios enfoques, como la medicina social, la epidemiología social, la antropología médica, la ecología humana $(12,13)$ y la iniciativa Una salud, también conocida como salud única o compartida y que consiste en resaltar la sinergia que existe al fomentar una mayor cooperación entre la salud humana, animal y ambiental (14).

La construcción por la equidad de bienes públicos nacionales e internacionales en salud - como los sistemas de salud, las instituciones de investigación en salud pública y entes intangibles como la promoción y la prevención, el control y la eliminación de enfermedades- son medios efectivos para la búsqueda de la equidad en salud. Representan la voluntad y la lucha ciudadana y en muchos casos resultan de la solidaridad entre pueblos y naciones.

\section{OBJETIVOS DE LA RAES A CORTO Y MEDIANO PLAZOS}

Compartir experiencias exitosas en el desarrollo de intervenciones, considerando la determinación y los determinantes sociales, para lograr respuestas participativas y comunitarias en salud. En las Américas, las mediciones en salud muestran la persistencia del cuadro de inequidad con relación al género, la identidad sexual, la edad, la etnicidad, el color de piel y la posición socioeconómica, características que están asociadas a la desigualdad y a la injusticia social y a la no realización de los derechos humanos (15-21). Muchos de los grandes problemas de salud pública se concentran en las zonas más pobres y desatendidas de las grandes ciudades (22) y en las comunidades indígenas y afrodescendientes (23).

Desde la RAES se estudia los sistemas de salud con énfasis en la atención primaria en salud (APS) y los servicios comunitarios dotados de acciones sociosanitarias, como los de Costa Rica y Cuba (Cuadro 1). En ambos países, la respuesta a la pandemia se apoya en la APS, que se ha activado para que siga funcionando con normalidad, porque el control de las cadenas de contacto de la transmisión de la COVID-19 se realiza a nivel comunitario y con la participación activa de la comunidad. Ambos casos muestran que las respuestas más eficaces a la pandemia provienen de los modelos de APS arraigados en la comunidad y no en los hospitales.

En Costa Rica, la información consignada de cada persona, familia y comunidad en el expediente digital ha permitido la valoración de riesgo de los pacientes crónicos, la entrega de medicamentos en el domicilio, la teleconsulta y el seguimiento por medios electrónicos. Asimismo, se creó una normativa según la cual a las mujeres embarazadas de bajo riesgo se las llama por teléfono y se verifica que han realizado los exámenes. Si existía o se detecta riesgo medio, la mujer debe acudir a una clínica del sistema de atención primaria y, si tiene riesgo alto, a un hospital. Para la atención de la COVID-19 se han fortalecido los hospitales regionales con equipos de protección personal y pruebas de COVID-19, y a cada persona que llega a un hospital 
CUADRO 1. Casos identificados por la Red de las Américas para la Equidad en Salud que contribuyen a la equidad en salud o que requieren acción

Compartir experiencias exitosas en el desarrollo de intervenciones, considerando la determinación y los determinantes sociales, para lograr respuestas participativas y comunitarias en salud

Se ha identificado a Cuba y Costa Rica como países en los cuales el sistema de salud pública está caracterizado por una alta coordinación y está basado en una APS equitativa, de gran capacidad resolutiva y articulada con la red hospitalaria $(29,30)$.

Analizar los impactos sanitarios, sociales, políticos, ambientales y económicos de la pandemia por COVID-19

Como la epidemia podría continuar, reactivarse o dar lugar a rebrotes, los esfuerzos de la RAES se orientan a lograr, en los ámbitos comunitario, nacional y regional, un entendimiento más profundo con énfasis en quienes tienen menor acceso a la salud y al bienestar, y en donde el confinamiento ha resultado no viable, atendible 0 claramente perjudicial para los sectores más vulnerables. La Red busca identificar qué cambios tendrían que introducirse — p. ej., en el manejo alimentario (31) 0 en la atención de otros problemas de salud (32) - para, frente a un hipotético nuevo rebrote, poder aplicar medidas que no amplíen la inequidad sino que la compensen y que disminuyan de manera efectiva el riesgo de enfermar y de morir. También se identificarán formas de respuesta desde la sociedad civil que han atendido vacíos o constituido importantes espacios de apoyo 0 crítica constructiva a las acciones gubernamentales no realizadas.

\section{Identificar los efectos de la atención de la COVID-19 en las poblaciones de mayor riesgo por su edad o por las condiciones de salud preexistentes}

En Costa Rica, la RAES ha identificado una estrategia para garantizar el acceso a los medicamentos para las enfermedades crónicas. La Caja Costarricense del Seguro Social firmó un convenio de cooperación público-privada según la cual se utilizan autos de alquiler, de correos y de los municipios con vehículos disponibles, y se aporta gasolina y choferes, para distribuir los medicamentos a domicilio y evitar los desplazamientos de la población. Este sistema de distribución incluye medicamentos para enfermedades crónicas, como antihipertensivos, insulina y antirretrovirales, así como anticonceptivos, pero se excluyen los medicamentos de alto riesgo. El programa ha sido muy bien valorado por la población.

\section{Examinar la situación de las fronteras y de los movimientos de población en la propagación de la pandemia y de sus efectos en las poblaciones migrantes}

La RAES propone monitorear la situación en la Amazonia, donde observamos el día a día difícil e injusto que se impone a las naciones indígenas, cuyos territorios están invadidos y cuyas poblaciones están siendo diezmadas por enfermedades como la COVID-19, a la cual son especialmente vulnerables $(33,34)$.

Proponer estrategias para asegurar el acceso a la atención integral de las mujeres gestantes con el fin de reducir el sufrimiento, la morbilidad y la mortalidad materna y neonatal

La RAES ha identificado el Hospital Monseñor Sanabria en Puntarenas (Costa Rica) como caso para mostrar la transformación, iniciada en 2015, de un modelo de atención biologista que no satisfacía a las usuarias hacia un modelo de atención centrado en la mujer, con perspectiva de género y enfoque de derechos.

Analizar vulneraciones de derechos humanos y del derecho a la salud de las poblaciones históricamente marginalizadas, incluyendo habitantes en situación de calle y otras comunidades que dependen de los espacios públicos y de la calle para sobrevivir

La RAES propone tres líneas de acción para trabajar a nivel callejero y a nivel de políticas de salud: 1) Coordinar una junta regional comunitaria que reúna voces comunitarias desde diferentes países en las Américas, incluyendo Colombia, Brasil, Argentina, Uruguay y México, donde existen movimientos de base por los derechos de los habitantes de calle, trabajadores sexuales y usuarios de drogas. Esta junta se reunirá tres veces al año para coordinar acciones regionales contra la violencia estatal y exclusión del sistema de salud que experimenta esta población. 2) Coordinar un sistema regional de denuncia comunitaria de negligencia del sistema de salud que operará en un formato de transmisión en vivo desde la página web de la RAES (con los puntos de contacto de la junta regional comunitaria como voceros de la denuncia). 3) Coordinar un sistema de respuesta que movilice actores de la política pública y las políticas de salud para catalizar respuestas inmediatas a los casos. Este sistema de denuncia comunitaria y acción en red regional desde la RAES es la manifestación de la investigación-acción participativa que informa nuestras acciones.

se le toma la temperatura y se evalúan los síntomas; en caso de sospecha, se la dirige a la emergencia para separarla del resto de las personas que acuden. Se designó un área del Centro Nacional de Rehabilitación, que cuenta con terapia respiratoria, para atender a las personas con COVID-19.

Cuba cuenta con la fortaleza del modelo de medicina familiar y la red de servicios integrales de la APS. Desde el inicio de la pandemia, desde un sistema de vigilancia epidemiológica comunitaria se inició la búsqueda de personas con síntomas y asintomáticas, y se llevó a cabo la vigilancia de los viajeros internacionales. Ello ha permitido que alrededor del 50\% de los casos confirmados se encontraran en un estadio asintomático (24). A los contactos de casos positivos se les aconsejó permanecer en cuarentena por 14 días en centros hospitalarios designados o en sus domicilios, con restricción de movimientos, controles desde las unidades de primer nivel para la APS y pruebas de PCR-TR entre el tercer y cuarto día. Las personas con síntomas se derivaron a centros hospitalarios con mayor nivel de resolución clínica. En ningún momento colapsaron los servicios de urgencia o las unidades de cuidados intensivos. Las autoridades gubernamentales implementaron otro grupo de intervenciones para conseguir un distanciamiento físico de la población en los diferentes estadios de la epidemia, con mayor énfasis en las comunidades con brotes locales de transmisión o mayor número de casos, así como la ejecución de campañas de información pública, todas ellas reconocidas como intervenciones efectivas para el control de la transmisión (25).

Otros países de la Región han implementado también estrategias exitosas. En Brasil, por ejemplo, la Fundación Oswaldo Cruz (FIOCRUZ) ha promocionado proyectos para la protección de las poblaciones más vulnerables ante la pandemia (26). Esta iniciativa movilizó una gran cantidad de acciones que, con un modesto financiamiento y el compromiso de una gran cantidad de personas y organizaciones, lograron innovar en la promoción de la protección sanitaria, la seguridad alimentaria, la educación, la protección social, la cultura y la atención pública en salud. FIOCRUZ también desarrolla y produce kits diagnósticos y medicinas, provee servicios clínicos a las personas con COVID-19, capacita al personal de salud en gran escala y avanza en los preparativos para el desarrollo, la producción y la distribución de una vacuna en América Latina y el Caribe.

La RAES también busca y promueve opciones viables y plantea alternativas de mediano y largo plazos para una mayor equidad con un sentido de urgencia, encontrando agentes de transformación que promuevan cambios a través de políticas y programas sociales y una práctica volcada a la promoción de la equidad en salud. Este sentido de urgencia se ha reforzado debido a la pandemia de COVID-19, que amenaza con revertir los logros obtenidos para cumplir las metas de la Agenda $2030(27,28)$. 
Analizar los impactos sanitarios, sociales, políticos, ambientales y económicos de la pandemia de COVID-19. La crisis ocasionada por la COVID-19 ha enfrentado a las Américas a una situación inédita. En casi toda la Región se suspendieron los servicios ambulatorios (excepto las emergencias) para problemas de salud no relacionados con la COVID-19 y se estableció el confinamiento obligatorio de la población en sus hogares, permitiendo solo salidas breves para abastecimiento de alimentos y con distancia física obligatoria, uso de mascarillas y otras medidas. Considerando el potencial impacto negativo de estas restricciones sobre la economía nacional y de cada hogar, algunos países ofrecieron paquetes de apoyo económico, intentando priorizar a la población más desatendida $(27,35,36)$.

En Perú, la RAES ha identificado que la experiencia de la epidemia y del confinamiento ha sido muy diversa dentro del país, y siempre más difícil entre la población dependiente de una economía informal de subsistencia (microcomercialización, limpieza o cuidado de vehículos o limpieza doméstica), mientras que la mayoría de quienes cuentan con empleos formales mantuvieron sus trabajos y salarios. La población más vulnerable con frecuencia carecía de cuentas bancarias, lo que retrasó en más de 45 días la transferencia de sus bonos de apoyo, y carecía también de fondos pensionarios o de la 'Compensación por Tiempo de Servicios' (que funciona como seguro de desempleo), fondos que en ambos casos se liberaron de manera parcial. Así pasaron hambre, salieron a buscar ingresos que les permitieran alimentar a sus familias y con frecuencia se infectaron, llegando algunos de ellos a estados críticos o a la muerte. Aunque dentro de sus mismos hogares hacinados no era fácil prevenir la infección de los familiares, muchos medios los culparon por su 'irresponsabilidad' al no 'quedarse en casa' $(37,38)$. Desde muy temprano en la epidemia en Perú se constituyó un núcleo de profesionales de la salud pública y de los aspectos sociales de la salud, conectados por una lista de WhatsApp, que tempranamente estableció contacto con el Ministerio de Salud y organizó reuniones virtuales semanales para discutir varios aspectos de la crisis, desarrollando documentos técnicos dirigidos al gobierno o emitiendo pronunciamientos que expresaban una crítica constructiva. Ahora establecido como la Plataforma Ciudadana para la Formulación de Políticas en Salud y Derechos Humanos, está articulando una visión a largo plazo centrada en una reforma integral del sistema de salud (39).

En México, la RAES colabora con el Grupo de Estudios sobre la Mujer Rosario Castellanos (GESMujer) en el área de prevención y atención de la violencia hacia las mujeres. Entre el inicio del período de aislamiento y mayo de 2020, la violencia de género creció un $26 \%$ en Oaxaca (40), problema que agrava la situación económica ya que la mayoría de las mujeres tienen trabajos de subsistencia. Desde el primer día de la cuarentena, GESMujer puso a disposición de las mujeres un número de asistencia remota atendido por psicólogas y asesoras legales, que ha canalizado numerosos casos de violencia sexual por las dificultades de acudir a solicitar atención médica ante el riesgo de contagio (40) y ha mantenido contacto con instancias gubernamentales que ofrecen otros servicios de asistencia a las víctimas de violencia. GESMujer, al reconocer que los efectos de la pandemia tendrán implicaciones en los temas de violencia y salud sexual y reproductiva que continuarán más allá del desconfinamiento, impulsará acciones en las áreas de prevención, atención y monitoreo de los servicios de salud y procuración de justicia. En materia de prevención y detección de señales de peligro de casos de violencia o de salud sexual y reproductiva, GESMujer realizará cápsulas en audio y video con contenidos multiculturales y traducción a tres lenguas indígenas mayoritarias con la colaboración de líderes indígenas formadas en la organización. En el área de atención, GESMujer continuará brindando asesoría psicológica y legal, con énfasis en violencia sexual y sus consecuencias, así como documentación de casos para conocer la atención brindada en los servicios públicos de salud y justicia y para realizar recomendaciones a instancias de gobierno y mejorar su respuesta a las mujeres.

Identificar los efectos de la atención de la COVID-19 en las poblaciones de mayor riesgo por su edad y por las condiciones de salud preexistentes. Ante el aumento del número de personas enfermas con COVID-19 en las Américas, la RAES participa en la activación y el fortalecimiento de los sistemas de protección, promoción y prevención para la población mayor de 60 años de zonas urbanas y rurales (Cuadro 1). Estos son los grupos de más alto riesgo cuando se consideran los casos hospitalizados y las defunciones, ya que su edad está vinculada con enfermedades crónicas preexistentes $(41,42)$. Desde la visión de la salud pública, la prevención y la identificación de factores de riesgo son necesarias para diseñar estrategias de actualización y capacitación del personal de salud y contar con el equipamiento adecuado e insumos suficientes en las unidades del primer nivel de atención (43). Asimismo, es necesario orientar y motivar la participación comunitaria de la población y de los sectores público y privado para atender integralmente la emergencia sanitaria desde la perspectiva de la determinación y los determinantes sociales de la salud. Desafortunadamente, en este grupo poblacional la inequidad en salud y la exclusión son los desafíos más importantes para la salud pública y el bienestar. Dar prioridad a los mayores de 60 años con la participación de los equipos de salud de la APS es un área de oportunidad que debe aprovecharse durante la pandemia con estrategias de prevención, promoción, información, educación y comunicación de los factores de riesgo de las comorbilidades para evitar contagios y reducir la demanda de servicios curativos en hospitales de segundo y tercer nivel, que tienen un mayor riesgo de contaminación y transmisión con el SARS-CoV-2 (44).

Examinar la situación de las fronteras y de los movimientos de población en la propagación de la pandemia y de sus efectos en las poblaciones migrantes. Existen situaciones especiales en territorios de frontera en que al mismo tiempo se observan solidaridad y dramas humanos de migración forzada por los cambios ambientales y la inseguridad humana. Con esta nueva amenaza a las naciones indígenas, en un momento en el cual los mecanismos de control están debilitados, queda amenazado también el patrimonio cultural de la región. La ONU ha realizado recomendaciones y establecido directrices para reconocer a las instituciones, autoridades y gobiernos representativos de los pueblos indígenas e incluir a sus representantes; proporcionar apoyo efectivo a las comunidades indígenas para detener la propagación de la COVID-19; mejorar el acceso y la gestión del agua potable y el saneamiento (en particular para los pueblos indígenas que viven en comunidades remotas); garantizar la disponibilidad de datos desagregados de los pueblos indígenas; obtener el consentimiento libre, previo e informado de los 
pueblos indígenas antes de iniciar cualquier programa o actividad; establecer actividades y programas de reconstrucción pospandemia dirigidos específicamente a los pueblos indígenas que apoyan los medios de vida tradicionales; e involucrar a los jóvenes indígenas (45).

En América Latina son muchos los factores que promueven la migración regional, y las difíciles condiciones económicas y sociales previas y posteriores a la pandemia hacen de esto un desafío creciente e injusto. La RAES establecerá vínculos para estudiar las respuestas a la pandemia en las fronteras de la Amazonia (Cuadro 1), de Centroamérica, y entre Haití y la República Dominicana.

Proponer estrategias para asegurar el acceso a la atención integral de las mujeres gestantes con el fin de reducir el sufrimiento, la morbilidad y la mortalidad materna y neonatal. Desde la RAES se realizan investigaciones para identificar las mejores prácticas en la atención a las mujeres gestantes y los retos a la atención digna y respetuosa y para analizar la contribución de la violencia obstétrica a la inequidad en los indicadores de salud materna y neonatal en distintos grupos de población de las Américas (Cuadro 1). El abuso verbal, el incumplimiento de los protocolos de atención, la mala relación entre mujeres y profesionales de salud, y las condiciones y limitaciones del sistema de salud son formas frecuentes de violencia obstétrica (46), un tema crítico pero desatendido que requiere un conocimiento preciso de su frecuencia e impacto (47). Para garantizar resultados óptimos en salud, las dimensiones clínicas y no clínicas de la violencia obstétrica deben ser entendidas y abordadas como impulsoras de la inequidad en la morbimortalidad materna y neonatal, ya que pueden generar una prestación de atención en salud deficiente o negligente $(23,46-48)$. Aunque una muerte materna se considera un evento raro en términos epidemiológicos, cada año en las Américas ocurren aproximadamente 8500 muertes, 23 cada día, generalmente como resultado de causas prevenibles (49). Estas muertes ocurren de forma desproporcionada en mujeres indígenas, afrodescendientes y quienes viven en condiciones de pobreza (21). Conocer experiencias exitosas y su impacto en la salud materna e infantil es una gran contribución al desarrollo de estrategias y programas para mejorar las prácticas de atención y las decisiones del personal de salud sobre los derechos de las mujeres y para animar a más mujeres a buscar asistencia calificada en el parto sin temor a la violencia.

Analizar vulneraciones de derechos humanos y del derecho a la salud de las poblaciones históricamente marginalizadas, incluyendo habitantes en situación de calle y otras comunidades que dependen de los espacios públicos y de la calle para sobrevivir. Para la RAES es una prioridad levantar las vivencias y las voces de las comunidades históricamente marginalizadas y excluidas socialmente que sobreviven en medio de la pobreza extrema, el abandono estatal y las barreras que bloquean, en múltiples niveles, el acceso al derecho a la salud (Cuadro 1). La comunidad en situación de calle incluye a habitantes de calle, trabajadores sexuales y vendedores ambulantes que dependen del diario "rebusque" para sobrevivir. Esta línea de acción está informada por los principios y praxis de la investigación-acción participativa, que entendemos como una filosofía de vida más que como un método de investigación (50). Esta filosofía recuerda la esencia del poder del pueblo y fija la mirada analítica de la Red hacia las calles, que entendemos como el cementerio de la pobreza extrema, donde todos los días mueren personas por actos de violencia policial, negligencia de los sistemas de salud y procesos de estigmatización que criminalizan a los cuerpos de la pobreza. Queremos resaltar el espacio político de la calle como eje socioespacial clave para nuestra agenda de investigación-acción participativa. Es a la calle adonde llegan millones de personas en las Américas para sobrevivir desde la exclusión y violencia de la informalidad. Durante la pandemia, la muerte de Alejandra "la Monocuco", una mujer trans afrodescendiente y trabajadora sexual que vivía con VIH en Bogotá, es un ejemplo trágico de violencia estatal que sucede todos los días en las Américas (51). Alejandra murió 40 minutos después de que una ambulancia, llamada por sus compañeras para atender una emergencia de salud, la abandonó. Como tantas personas que sobreviven en medio de la pobreza extrema y de la violencia estructural y policial, Alejandra forma parte de los millones de personas en las Américas que han llegado a las calles para luchar por su vida. Este momento histórico es una oportunidad para catalizar cambios institucionales que garanticen como mínimo una vida digna para estas comunidades.

\section{SUMANDO ESFUERZOS}

La RAES mantiene alianzas con entidades académicas y no gubernamentales, con sociedades científicas y con organizaciones internacionales, como la Universidad de Costa Rica, el Grupo Colaborador para la Equidad en Salud de la Universidad de Tulane, el Grupo de Estudios sobre la Mujer Rosario Castellanos, la Universidad Peruana Cayetano Heredia, la Fundación Oswaldo Cruz, el Instituto de Medicina Tropical Pedro Kourí, la Universidad de California en Los Ángeles, Investigación en Salud y Demografía, la Academia Nacional de Medicina de México, la Organización Panamericana de la Salud, la Asociación de Escuelas y Programas de Salud Pública, la Alianza Latinoamericana de Salud Global, el Instituto de Equidad en Salud del University College London y la Fundación Robert Wood Johnson, además de organismos multilaterales.

El trabajo en red de los miembros de la RAES permite agilizar la investigación concertada con compromiso social y con planes de acción para el cambio social en aras de fomentar la inclusión social, la equidad en salud y los derechos humanos en las Américas. Los vínculos que la RAES establece con otras instituciones permiten desarrollar planes de formación y actualización de los recursos humanos en salud y monitorear y evaluar las intervenciones encaminadas a lograr la equidad en salud.

Contribución de los autores. Todos los autores concibieron el manuscrito y contribuyeron con su redacción, revisión y aprobación de la versión final.

\section{Conflictos de intereses. Ninguno declarado.}

\section{Financiación. Ninguna declarada.}

Declaración. Las opiniones expresadas en este manuscrito son únicamente responsabilidad de los autores y no reflejan necesariamente las de la Revista Panamericana de Salud Pública o la Organización Panamericana de la Salud. 


\section{REFERENCIAS}

1. Rodriguez MA, Marmot MG, Salgado de Snyder VN, Galvao LAC, Avellaneda X, Saenz M R, et al. The Transformative Potential of Strategic Partnerships to Form a Health Equity Network of the Americas. Ethn Dis. 2019;29(Suppl 1):153-8.

2. Almeida-Filho N. A problemática teórica da determinação social da saude (nota breve sobre desigualdades em saude como objeto de conhecimento). Saude em Debate. 2009;33(83):349-70.

3. Organización Panamericana de la Salud. Sociedades justas: equidad en la salud y vida digna. Informe de la Comisión de la Organización Panamericana de la Salud sobre Equidad y Desigualdades en Salud en las Américas. Washington, DC: Organización Panamericana de la Salud; 2019. Disponible en: iris.paho.org/handle/10665.2/51615.

4. Marmot M. Social determinants of health inequalities. Lancet. 2005;365(9464):1099-104.

5. Plough AL, editor. Advancing Health and Well-Being: Using Evi dence and Collaboration to Achieve Health Equity. New York: Oxford University Press; 2018.

6. Organización Mundial de la Salud, Gobierno de Australia Meridional. Declaración de Adelaida sobre la Salud en Todas las Políticas. Adelaida: Organización Mundial de la Salud y Gobierno de Australia Meridional; 2010. Disponible en: https://bit.ly/2AtroTf.

7. Whitmee S, Haines A, Beyrer C, Boltz F, Capon AG, de Souza Dias $\mathrm{BF}$, et al. Safeguarding human health in the Anthropocene epoch: report of The Rockefeller Foundation-Lancet Commission on planetary health. Lancet. 2015;386(10007):1973-2028.

8. Naciones Unidas. Resolución A/RES/70/1: Transformar nuestro mundo: la Agenda 2030 para el Desarrollo Sostenible. Nueva York: Organización de las Naciones Unidas; 2015. Disponible en: https:/ / undocs.org/es/A/RES/70/1.

9. Naciones Unidas. Agenda 2030 sobre el Desarrollo Sostenible. Nueva York: Organización de las Naciones Unidas; 2015. Disponible en: https: / / www.un.org/sustainabledevelopment/es/.

10. Whitehead $M$. The concepts and principles of equity and health. Int J Health Serv. 1992;22(3):429-45.

11. Braveman P. Health disparities and health equity: concepts and measurement. Annu Rev Public Health. 2006;27:167-94.

12. Castro A. Health and inequality. En: Callan H, editor. The International Encyclopedia of Anthropology. Hoboken, NJ: Wiley-Blackwell; 2018.

13. Krieger N. A critical research agenda for social justice and public health: an ecosocial proposal. En: B L, editor. Social Injustice and Public Health, 3rd edition. New York: Oxford University Press; 2019.

14. Amuasi JH, Lucas T, Horton R, Winkler AS. Reconnecting for our future: The Lancet One Health Commission. Lancet. 2020;395(10235):1469-71.

15. Dmytraczenko T, Almeida G, editors. Toward Universal Health Coverage and Equity in Latin America and the Caribbean. Evidence from Selected Countries. Washington, DC: The World Bank; 2015.

16. Etienne CF. Equity in health systems. Rev Panam Salud Publ. 2013;33(2):79-80.

17. Cardona D, Acosta LD, Bertone CL. Inequidades en salud entre países de Latinoamérica y el Caribe (2005-2010) [Inequities in health among Latin American and Caribbean countries (2005-2010)]. Gaceta sanitaria. 2013;27(4):292-7.

18. de Andrade LO, Pellegrini Filho A, Solar O, Rigoli F, de Salazar LM, Serrate PC, et al. Social determinants of health, universal health coverage, and sustainable development: case studies from Latin American countries. Lancet. 2015;385(9975):1343-51.

19. Cotlear D, Gómez-Dantés O, Knaul F, Atun R, Barreto ICHC, Cetrángolo $\mathrm{O}$, et al. Overcoming social segregation in health care in Latin America. Lancet. 2015;385(9974):1248-59.

20. Barros AJ, Victora CG. Measuring coverage in MNCH: determining and interpreting inequalities in coverage of maternal, newborn, and child health interventions. Plos Med. 2013;10(5):e1001390.

21. UNICEF y Tulane University. Health Equity Report 2016: analysis of reproductive, maternal, newborn, child and adolescent health inequities in Latin America and the Caribbean to inform policy making; 2016.

22. Diez Roux AV, Slesinski SC, Alazraqui M, Caiaffa WT, Frenz P, Jordan Fuchs R, et al. A Novel International Partnership for Actionable Evidence on Urban Health in Latin America: LAC-Urban Health and SALURBAL. Glob Chall. 2019;3(4):1800013.
23. Castro A, Savage V, Kaufman H. Assessing equitable care for Indigenous and Afrodescendant women in Latin America. Rev Panam Salud Publica. 2015;38(2):96-109.

24. Mas Bermejo P, Vidal Ledo MJ, Baldoquín Rodríguez W, Seuc Jo AH, Guinovart Díaz R, Noriega Bravo V. Lucha anti epidémica en la COVID-19 en Cuba. Organización de la investigación epidemiológica. Información para Directivos de la Salud. 2020;32.

25. Anderson RM, Heesterbeek H, Klinkenberg D, Hollingsworth TD. How will country-based mitigation measures influence the course of the COVID-19 epidemic? Lancet. 2020;395(10228):931-4.

26. Gomez L. Covid-19: Fiocruz lança ações de apoio a populações vulneráveis. Rio de Janeiro: FIOCRUZ; 2020. Disponible en: https:// portal.fiocruz.br/noticia/covid-19-fiocruz-lanca-acoes-de-apoio -populacoes-vulneraveis.

27. Castro A. Desafíos de la pandemia de COVID-19 en la salud de la mujer, de la niñez y de la adolescencia en América Latina y el Caribe. PNUD LAC C19 PDS No. 19. Nueva York: Programa de Naciones Unidas para el Desarrollo; 2020. Disponible en: https:// www.latinamerica.undp.org/content/rblac/es/home/library/ crisis_prevention_and_recovery/desafios-de-la-pandemiade-covid-19-en-la-salud-de-la-mujer--de-.html.

28. Comisión Económica para América Latina y el Caribe. La Agenda 2030 para el Desarrollo Sostenible en el nuevo contexto mundial y regional. Escenarios y proyecciones en la presente crisis. Santiago de Chile: CEPAL; 2020. Disponible en: https://repositorio.cepal. org/bitstream/handle/11362/45336/6/S2000208_es.pdf.

29. Organización Panamericana de la Salud. La salud en las Américas. Washington DC: Organización Panamericana de la Salud; 2017. Disponible en: https://www.paho.org/salud-en-las-americas-2017/? lang=es:

30. Banco Interamericano de Desarrollo. El impacto del COVID19 en las economías de la región. La pandemia de COVID-19 en Centroamérica, Haití, México, Panamá y República Dominicana. Washington, DC.: BID; 2020.

31. FAO, CEPAL. Análisis de Respuestas en América Latina y el Caribe ante el COVID-19 en los sistemas alimentarios $\mathrm{N}^{\circ} 2$. Santiago de Chile: Comisión Económica para América Latina y el Caribe; 2020. Disponible en: https://www.cepal.org/es/publicaciones/ 45524-analisis-respuestas-america-latina-caribe-efectos-covid19-sistemas-alimentarios.

32. Organización de las Naciones Unidas. Es vital que la respuesta de los países de América al coronavirus incluya tratar las enfermedades crónicas. Noticias ONU, 26 de mayo 2020. Disponible en: https://news.un.org/es/story/2020/05/1474992.

33. Pontes AL, Alarcon DF, Kaingang JD, Santos RV. Vulnerabilidades, impactos e o enfrentamento ao Covid-19 no contexto dos povos indígenas: reflexões para a ação. Rio de Janeiro: FIOCRUZ; 2020. Disponible en: https://bit.ly/38FxtZD

34. Fondo para el Desarrollo de los Pueblos Indígenas de América Latina y El Caribe. Los Pueblos Indígenas ante la pandemia del COVID-19. Primer Informe Regional. La Paz: FILAC; 2020. Disponible en: https:/ /indigenascovid19.red/archivos/13326.

35. Romo R. Latinoamérica responde al coronavirus: los países que han fallado (y acertado) en su respuesta al covid-19. CNN en Español, 29 de mayo. 2020. Disponible en: https://cnnespanol. cnn.com/2020/05/29/latinoamerica-responde-al-coronavirus-lospaises-que-han-fallado-y-acertado-en-su-respuesta-al-covid-19/.

36. Comisión Económica para América Latina y el Caribe. Sectores y empresas frente al COVID-19: emergencia y reactivación. Santiago de Chile: CEPAL; 2020. Disponible en: https://www.cepal.org/ es / publicaciones / 45734-sectores-empresas-frente-al-covid-19emergencia-reactivacion.

37. Benavente L. Por qué el Perú es el país con más casos por millón de covid-19 en América Latina. RPP Noticias, 18 de mayo 2020. Disponible en: https://rpp.pe/peru/actualidad/ por-que-peru-es-el-pais-con-mas-casos-por-millon-de-covid-19-enamerica-latina-noticia-1266444.

38. La Torre A, Ghezzi P, Segura A. ¿Qué hacemos cuando el martillo no chanca? La República, 19 de abril 2020. Disponible en: https:// larepublica.pe/sociedad/2020/04/19/coronavirus-en-peru-quehacemos-cuando-el-martillo-no-chanca-la-curva-del-covid-19/. 
39. Ciriaco M. Una plataforma para defender el derecho a la salud de los peruanos. Salud con Lupa, 9 de julio 2020. Disponible en: https: / / saludconlupa.com/entrevistas/una-plataforma-para-defenderel-derecho-la-salud-de-los-peruanos/.

40. GESMujer. Grupo de estudios sobre la mujer Rosario Castellanos. Disponible en: https:/ /www.gesmujer.org/web/. 2020.

41. Pueyo T. Coronavirus: The Hammer and the Dance: What the Next 18 Months Can Look Like. Medium, 19 de marzo. 2020. Disponible en: https://medium.com/@tomaspueyo/coronavirusthe-hammerand-the-dance-be9337092b56.

42. Organización Mundial de la Salud. Joint Mission. Report of the WHO-China Joint Mission on Coronavirus Disease 2019 (COVID19). Ginebra: Organización Mundial de la Salud; 2020. Disponible en: https://www.who.int/docs/default-source/coronaviruse/whochina-joint-mission-on-covid-19-final-report.pdf.

43. Ferguson NM, Laydon D, Nedjati-Gilani G, Imai N, Ainslie K, Baguelin $\mathrm{M}$, et al. Reporte 9: Impacto de las intervenciones no farmacéuticas (INF) para reducir la mortalidad y demanda en los sistemas de salud frente a COVID-19 [Impact of non-pharmaceutical interventions (NPIs) to reduce COVID-19 mortality and healthcare demand], Spanish translation. Imperial College London, 16 de marzo 2020. Disponible en: https:/ / doi.org/10.25561/77482.

44. Ye G, Lin H, Chen S, Wang S, Zeng Z, Wang W, et al. Environmental contamination of SARS-CoV-2 in healthcare premises. J Infect. 2020;81(2):e1-e5.

45. Naciones Unidas. The Impact of COVID-19 on Indigenous Peoples. Nueva York: Naciones Unidas; 2020. Disponible en: https://bit. ly/2ZXCbOe.
46. Castro A, Savage V. Obstetric Violence as Reproductive Governance in the Dominican Republic. Med Anthropol. 2019;38(2):123-36.

47. Castro A. Witnessing Obstetric Violence during Fieldwork: Notes from Latin America. Health Hum Rights. 2019;21(1):103-11.

48. Savage V, Castro A. Measuring mistreatment of women during childbirth: a review of terminology and methodological approaches. Reprod Health. 2017;14(1):138.

49. OMS, UNICEF, UNFPA, Banco Mundial, División de Población de las Naciones Unidas. Trends in Maternal Mortality: 2000 to 2017. Ginebra: Organización Mundial de la Salud; 2019. Disponible en: https://www.who.int/reproductivehealth/publications/ maternal-mortality-2000-2017/en/.

50. Fals Borda O, Anisur Rahman M. Action and Knowledge: Breaking the Monopoly with Participatory Action Research. New York: Apex Press; 1991.

51. ¡Queremos la verdad! \#JusticiaParaAlejandra. El Espectador, 6 de junio 2020. Disponible en: https://www.elespectador.com/ noticias/nacional/muerte-de-alejandra-monocuco/.

Manuscrito recibido el 18 de agosto de 2020. Aceptado para su publicación, tras revisión, el 15 de septiembre de 2020.

\section{The Health Equity Network of the Americas: inclusion, commitment, and action}

ABSTRACT The Health Equity Network of the Americas (HENA) is a multidisciplinary network that promotes knowledge sharing and intersectoral action for equity in health and human rights in the Americas. The objectives of HENA are: 1) to share successful experiences in the development of interventions, considering the social determinants and determination of health, to achieve participatory and community-based health responses; 2) to analyze the health, social, political, environmental and economic impacts of the COVID-19 pandemic; 3) to identify the effects of pandemic care on populations most at risk because of their age and pre-existing health conditions; 4) examine the situation at borders and population movements in the spread of the pandemic and its effects on migrant populations; 5) propose strategies to ensure access to comprehensive care for pregnant women in order to reduce maternal and neonatal suffering, morbidity, and mortality; and 6) analyze violations of human rights and the right to health of historically marginalized populations, including street dwellers and other communities that depend on public spaces and the street for survival. The analytical and intervention models for health equity at HENA are based on various approaches, including social medicine, social epidemiology, medical anthropology, human ecology, and One health.

Keywords Health equity; One health; intersectoral collaboration; social determinants of health; Americas. 